\title{
ALMA reveals the feeding of the Seyfert 1 nucleus in NGC 1566
}

\author{
F. Combes ${ }^{1}$, S. García-Burillo ${ }^{2}$, V. Casasola ${ }^{3}$, L. K. Hunt ${ }^{4}$, M. Krips ${ }^{5}$, A. J. Baker ${ }^{6}$, F. Boone ${ }^{7}$, A. Eckart ${ }^{8}$, I. Marquez ${ }^{9}$, \\ R. Neri ${ }^{5}$, E. Schinnerer ${ }^{10}$, and L. J. Tacconi ${ }^{11}$
}

1 Observatoire de Paris, LERMA, CNRS UMR8112, 61 Av. de l'Observatoire, 75014 Paris, France

e-mail: francoise.combes@obspm.fr

2 Observatorio Astronómico Nacional (OAN)-Observatorio de Madrid, Alfonso XII 3, 28014-Madrid, Spain

3 INAF - Istituto di Radioastronomia \& Italian ALMA Regional Centre, via Gobetti 101, 40129 Bologna, Italy

4 INAF - Osservatorio Astrofisico di Arcetri, Largo E. Fermi 5, 50125 Firenze, Italy

5 IRAM, 300 rue de la Piscine, Domaine Universitaire, 38406 Saint Martin d'Hères, France

6 Dep. of Physics \& Astronomy, Rutgers, the State University of New Jersey, 136 Frelinghuysen road, Piscataway NJ 08854, USA

7 CNRS, IRAP, 9 Av. colonel Roche, BP 44346, 31028 Toulouse Cedex 4, France

8 I. Physikalisches Institut, Universität zu Köln, Zülpicher Str. 77, 50937 Köln, Germany

9 Instituto de Astrofísica de Andalucía (CSIC), Apdo 3004, 18080 Granada, Spain

10 Max-Planck-Institut für Astronomie (MPIA), Königstuhl 17, 69117 Heidelberg, Germany

11 Max-Planck-Institut für extraterrestrische Physik, Giessenbachstr. 1, 85748 Garching bei München, Germany

Received 15 January 2014 / Accepted 10 March 2014

\section{ABSTRACT}

\begin{abstract}
We report ALMA observations of $\mathrm{CO}(3-2)$ emission in the Seyfert 1 galaxy NGC 1566, at a spatial resolution of 25 pc. Our aim is to investigate the morphology and dynamics of the gas inside the central kpc, and to probe nuclear fueling and feedback phenomena. NGC 1566 has a nuclear bar of $1.7 \mathrm{kpc}$ radius and a conspicuous grand design spiral starting from this radius. The ALMA field of view, of diameter $0.9 \mathrm{kpc}$, lies well inside the nuclear bar and reveals a molecular trailing spiral structure from 50 to $300 \mathrm{pc}$ in size, which is contributing to fuel the nucleus, according to its negative gravity torques. The spiral starts with a large pitch angle from the center and then winds up in a pseudo-ring at the inner Lindblad resonance (ILR) of the nuclear bar. This is the first time that a trailing spiral structure is clearly seen driving the gas inwards inside the ILR ring of the nuclear bar. This phenomenon shows that the massive central black hole has a significant dynamical influence on the gas, triggering its fueling. The gaseous spiral is well correlated with the dusty spiral seen through extinction in HST images, and also with a spiral feature emitting $0.87 \mathrm{~mm}$ continuum. This continuum emission must come essentially from cold dust heated by the interstellar radiation field. The $\mathrm{HCN}(4-3)$ and $\mathrm{HCO}^{+}(4-3)$ lines were simultaneously mapped and detected in the nuclear spiral. The $\mathrm{HCO}^{+}(4-3)$ line is 3 times stronger than the $\mathrm{HCN}(4-3)$, as expected when star formation excitation dominates over active galactic nucleus (AGN) heating. The $\mathrm{CO}(3-2) / \mathrm{HCO}^{+}(4-3)$ integrated intensity ratio is $\sim 100$. The molecular gas is in remarkably regular rotation, with only slight non-circular motions at the periphery of the nuclear spiral arms. These perturbations are quite small, and no outflow nor AGN feedback is detected.
\end{abstract}

Key words. galaxies: active - galaxies: individual: NGC 1566 - galaxies: ISM - galaxies: kinematics and dynamics galaxies: nuclei - galaxies: spiral

\section{Introduction}

An astrophysical problem that has seen much progress in recent years is understanding how active galactic nuclei (AGN) are fueled in galaxies, and how the energy generated by the active nucleus can in turn regulate gas accretion (e.g. Croton et al. 2006; Sijacki et al. 2007). This has important implications for the co-evolution of galaxies and black holes, which is observed through the now well established $\mathrm{M}-\sigma$ relation (e.g., Gültekin et al. 2009). Driving sufficient gas towards the center to sustain nuclear activity requires the removal of angular momentum from the gas, and therefore the creation of large nonaxisymmetries. The invoked dynamical mechanisms depend on the scale in question: at $10 \mathrm{kpc}$ scales, torques are produced by galaxy interactions and mergers (e.g., Hopkins et al. 2006; di Matteo et al. 2008); at kpc scales, bar instabilities, either internally driven by secular evolution or triggered by companions, can first feed a central starburst and then fuel the mas-

* Based on observations carried out with ALMA in cycle 0. sive black hole (García-Burillo et al. 2005). At 300 pc scales, the "bars within bars" scenario (e.g., Shlosman et al. 1989), together with $m=1$ instabilities, takes over as a dynamical mechanism; however, our IRAM-PdBI observational program NUclei of GAlaxies (NUGA) has revealed smoking-gun evidence of AGN fueling only in one third of the galaxies observed (GarcíaBurillo \& Combes 2012). This might be due to nuclear star formation or AGN feedback driving gas outflows that stop the fueling; galaxies will then undergo successive periods of fueling and starvation, and might be found in a feeding phase at $300 \mathrm{pc}$ scales only one third of the time. Other mechanisms could contribute to the fueling, especially towards the center of galaxies: viscous torques, from dense gas in regions of large shear, or dynamical friction of massive clouds against the old bulge stars (e.g. Combes 2002; Jogee 2006). At the radii of interest, though, these are slower than gravity torques, when present.

To go beyond these scales and explore the molecular ISM $30 \mathrm{pc}$ to $300 \mathrm{pc}$ scales in local AGN host galaxies, we have undertaken an observing campaign with ALMA, the only instrument able to probe the molecular emission with enough spatial 
resolution and sensitivity. Observations and simulations are advancing together, since it is only now that we can simulate coherently these scales with successive zoom-in re-simulations (e.g., Hopkins \& Quataert 2010; Renaud et al. 2013). At 30 pc scales, simulations suggest fueling involves a cascade of dynamical instabilities ( $m=2, m=1$ ), and the formation of a thick gas disk similar to a torus, subject to bending and warping instabilities (Hopkins et al. 2012). Since the gas concentration becomes large, the gas is highly unstable, clumpy and turbulent. Dynamical friction can then drive these massive clumps to the nucleus. Simulations reveal episodic feeding with 10 Myr timescales $(10 \mathrm{pc})$ or even lower $(0.1 \mathrm{pc})$. These fueling episodes are then quenched by either star formation winds or AGN outflows.

Recently, high velocity outflows have been discovered in the molecular gas of nearby AGN (Feruglio et al. 2010; Sturm et al. 2011; Alatalo et al. 2011; Dasyra \& Combes 2012). Outflows have been traced for a long time in ionized or atomic gas (Rupke et al. 2005; Riffel \& Storchi-Bergmann 2011). With our ALMA cycle 0 data, we have been able to confirm that molecular outflows are also seen in low-luminosity AGN. The outflow revealed in the Seyfert 2 NGC 1433 is the least massive molecular outflow ever seen in galaxy nuclei (Combes et al. 2013). In the prototypical Seyfert 2 NGC 1068, a clear molecular ouflow has also been detected, entrained by the AGN radio jets (Krips et al. 2011; García-Burillo et al. 2010, and in prep.).

These high angular resolution results have shown that fueling and feedback phases can occur simultaneously. Gas is inflowing in the plane, with some also outflowing along the minor axis at some angle with the plane. In NGC 1433, the CO map reveals a nuclear gaseous spiral structure inside the $460 \mathrm{pc}$-radius nuclear ring encircling the nuclear stellar bar. The nuclear spiral winds up in a pseudo-ring at $\sim 200 \mathrm{pc}$ radius, which might correspond to the inner Lindblad resonance (ILR). In spite of the presence of gas in rings at both outer and inner ILRs, some gas is finding its way to the center. In both of these Seyfert 2 galaxies, dust continuum emission is detected in a small nuclear disk, which might be interpreted as the molecular torus (Combes et al. 2013). In NGC 1068, the AGN is clearly off-center with respect to the torus, implying an $m=1$ perturbation (García-Burillo et al., in prep.).

In this paper, we present ALMA cycle 0 observations in the $\mathrm{CO}(3-2)$ line of the Seyfert 1 NGC 1566, with a spatial resolution of $\sim 25 \mathrm{pc}$. Its proximity $(10 \mathrm{Mpc})$ and small inclination of $35^{\circ}$ make NGC 1566 an ideal target to test and refine scenarios of AGN feeding and feedback, and discover new phenomena controlling gas structures and dynamics within $100 \mathrm{pc}$, where the dynamical time-scale is smaller than $3 \mathrm{Myr}$ (for $V_{\text {rot }} \sim$ $200 \mathrm{~km} \mathrm{~s}^{-1}$ ). In the next subsection, all relevant characteristics of NGC 1566 are described. Observations are detailed in Sect. 2 and results in Sect. 3. The interpretation in term of torques is discussed in Sect. 4, and conclusions are drawn in Sect. 5.

\subsection{NGC 1566}

NGC 1566, a nearly face-on spiral galaxy, is the brightest member of the Dorado group (Agüero et al. 2004; Kilborn et al. 2005). It has an intermediate strength bar type (SAB), and two strongly contrasted spiral arms, emanating from the bar and winding up in an outer pseudo ring. The remarkable granddesign spiral has inspired special studies of its logarithmic arm structure (Elmegreen \& Elmegreen 1990; Korchagin et al. 2000). There are two sets of spiral arms (see Fig. 1): the first set starts at the end of the nuclear bar of about $35^{\prime \prime}=1.7 \mathrm{kpc}$ in radius, better seen in the near infrared (Hackwell \& Schweizer 1983).

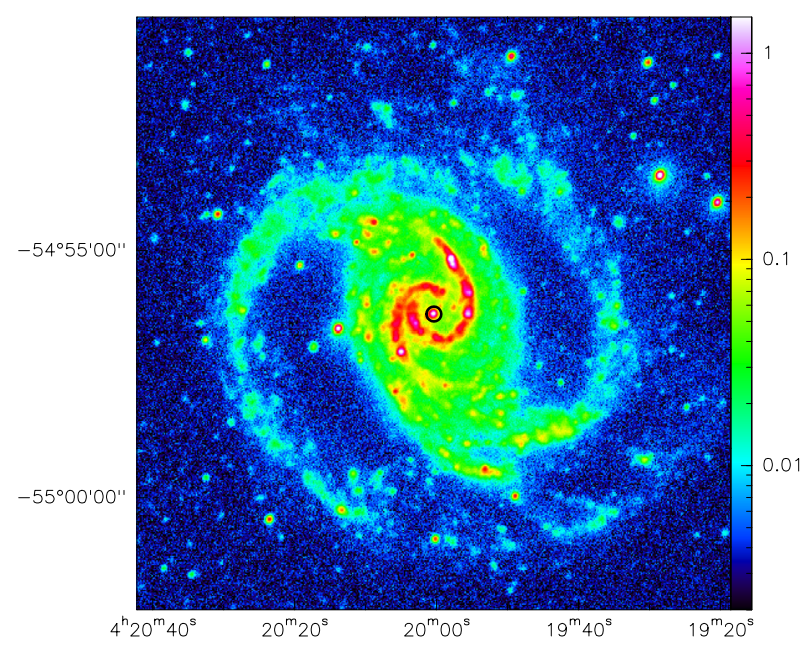

Fig. 1. GALEX-NUV image of NGC 1566, showing the two sets of spiral arm structures at large scales. The ALMA field of view ( $9^{\prime \prime}$ radius) is shown as the black circle in the center. The image is a square of $12^{\prime}$ size. The color scale is in log.

These arms then wind up in a ring, with kinks and a circle of star formation, identified as the corotation of the spiral pattern (Elmegreen \& Elmegreen 1990). A second weaker set of spiral arms then winds up in an outer pseudo ring, identified as the outer Lindblad resonance. The inner Lindblad resonance of the spiral might correspond to the end of the nuclear bar, and therefore the corotation of the bar. Inside this ILR, there is a deficiency of HII regions (Comte \& Duquennoy 1982).

NGC 1566 is a low-luminosity AGN, classified as Seyfert, although its precise position between Seyfert 1 and 2 varies in the literature. More specifically, its nuclear spectrum varies from Seyfert 1 in its most active phases to Seyfert 2 at minimum activity (Alloin et al. 1985). It has many characteristics of Seyfert 1 galaxies/AGN: a bright central point-like source (Malkan et al. 1998), relatively broad [FeII] line emission in the Broad Line Rregion (Reunanen et al. 2002), characteristic variability on scales of months (Alloin et al. 1986), and an $\mathrm{X}$-ray point-source nuclear luminosity between $2-10 \mathrm{keV}$ of $7 \times 10^{40} \mathrm{erg} / \mathrm{s}$ (Levenson et al. 2009). The nucleus is known to be variable from X-rays to IR bands (Alloin et al. 1986; Baribaud et al. 1992; Glass 2004). These variations have been interpreted as instabilities in a thin accretion disk (Abramowicz et al. 1986). The mass of the NGC 1566 black hole has been estimated to be $8.3 \times 10^{6} M_{\odot}$ by the $\mathrm{M}-\sigma$ relation and the measured stellar velocity dispersion at the center (Woo \& Urry 2002). From its bolometric luminosity, its Eddington ratio can be estimated as 0.096 (cf. Table 1). HST [OIII] images show a one-sided ionization cone towards the south-east (Schmitt \& Kinney 1996). VISIR images (Reunanen et al. 2010) reveal a dominant central unresolved source with size $F W H M<15$ pc at $11.8 \mu \mathrm{m}$. Both large-scale and nuclear radio emission is seen in NGC 1566 (Harnett 1984, 1987). The atomic gas (HI) dynamics have been investigated with the ATCA interferometer by Walsh (priv. comm.), with a spatial resolution of $\sim 30^{\prime \prime}$. There is a $30^{\prime \prime} \times 50^{\prime \prime}$ HI hole towards the center, where the gas becomes mostly molecular. The $\mathrm{CO}(1-0)$ and (2-1) lines have been mapped by Bajaja et al. (1995) with the SEST telescope, and are detected out to $60^{\prime \prime}$ radius. Excited molecular hydrogen has been detected through its ro-vibrational lines at $2 \mu \mathrm{m}$ (Reunanen et al. 2002). The $\mathrm{H}_{2}$ excitation is not by UV fluorescence but through thermal UV heating. The contribution from star formation is less 
Table 1. Basic data for the galaxy NGC 1566.

\begin{tabular}{lcc}
\hline \hline Parameter & Value $^{b}$ & Reference \\
\hline$\alpha_{\mathrm{J} 2000}{ }^{a}$ & $04^{\mathrm{h}} 20^{\mathrm{m}} 00.42^{\mathrm{s}}$ & $(1)$ \\
$\delta_{\mathrm{J} 2000{ }^{a}}$ & $-54^{\circ} 56^{\prime} 16.1^{\prime \prime}$ & $(1)$ \\
$V_{\text {hel }}$ & $1504 \mathrm{~km} \mathrm{~s}^{-1}$ & $(1)$ \\
RC3 Type & $\mathrm{SAB}(\mathrm{s}) \mathrm{bc}$ & $(1)$ \\
Nuclear Activity & Seyfert 1 & $(2)$ \\
Inclination & $35^{\circ} 0$ & $(3)$ \\
Position Angle & $44^{\circ} \pm 1^{\circ}$ & $(3)$ \\
Distance & $10 \mathrm{Mpc}\left(1^{\prime \prime}=48 \mathrm{pc}\right)$ & $(1)$ \\
$L_{B}$ & $8.8 \times 10^{9} L_{\odot}$ & $(4)$ \\
$M_{\mathrm{HI}}$ & $3.5 \times 10^{9} M_{\odot}$ & $(5)$ \\
$M_{\mathrm{H}}$ & $1.3 \times 10^{9} M_{\odot}$ & $(6)$ \\
$M_{\mathrm{dust}}(60$ and $100 \mu \mathrm{m})$ & $3.7 \times 10^{6} M_{\odot}$ & $(7)$ \\
$L_{\mathrm{FIR}}$ & $6.5 \times 10^{9} L_{\odot}$ & $(7)$ \\
$M_{\mathrm{BH}}$ & $8.3 \times 10^{6} M_{\odot}$ & $(2)$ \\
$L_{\mathrm{bol}}$ & $1.0 \times 10^{44} \mathrm{erg} / \mathrm{s}$ & $(2)$ \\
$\alpha_{\mathrm{J} 2000}{ }^{c}$ & $04^{\mathrm{h}} 20^{\mathrm{m}} 00.39^{\mathrm{s}}$ & New center \\
$\delta_{\mathrm{J} 2000}{ }^{c}$ & $-54^{\circ} 56^{\prime} 16.6^{\prime \prime}$ & New center \\
\hline
\end{tabular}

Notes. ${ }^{a}\left(\alpha_{\mathrm{J} 2000}, \delta_{\mathrm{J} 2000}\right)$ is the phase tracking center of our ${ }^{12} \mathrm{CO}$ interferometric observations. ${ }^{(b)}$ Luminosity and mass values extracted from the literature have been scaled to the distance of $D=10 \mathrm{Mpc} .{ }^{(c)}$ New adopted center, coinciding with the continuum peak (see Sect. 3.2).

References. (1) NASA/IPAC Extragalactic Database (NED, http:// nedwww.ipac. caltech.edu/); (2) Woo \& Urry (2002); (3) Agüero et al. (2004); (4) HyperLeda; (5) Reif et al. (1982); (6) Bajaja et al. (1995); (7) IRAS Catalog.

than $10 \%$, and the dominant $\mathrm{H}_{2}$ excitation mechanism is collisional via shocks. The derived $\mathrm{H}_{2}$ masses observed out to $2^{\prime \prime}$ from the center are $90 M_{\odot}$ along the ionized cone and $110 M_{\odot}$ perpendicular to it.

The distance of NGC 1566 is still subject to large uncertainty. The measured heliocentric velocity of $1504 \mathrm{~km} \mathrm{~s}^{-1}$, correcting for the solar motion inside the Local Group and accounting for the Virgocentric flow, yields a distance of $17.8 \mathrm{Mpc}$ (for a Hubble constant of $70 \mathrm{~km} \mathrm{~s}^{-1} \mathrm{Mpc}-1$ ). However, when the Tully-Fisher relation is used as distance indicator, the distance is found to be $6.5 \mathrm{Mpc}$ (e.g. Hyperleda). For the higher distance, the gas to dynamical mass ratio is unrealistically high, favoring lower values. In the present work, we adopt the median value given by NED, i.e. $D=10 \mathrm{Mpc}$, for which $1^{\prime \prime}=48$ pc.

\section{Observations}

The observations were carried out on October 22, 2012 with the ALMA array in cycle 0, using 23 antennae, under the project ID 2011.0.00208.S (PI: Combes). NGC 1566 was observed with Band 7 simultaneously in $\mathrm{CO}(3-2), \mathrm{HCO}^{+}(4-3)$, $\mathrm{HCN}(4-3)$, and continuum. The corresponding sky frequencies were $344.09 \mathrm{GHz}, 354.98 \mathrm{GHz}, 352.76 \mathrm{GHz}$, and $342.80 \mathrm{GHz}$, respectively. The observations were done in 2 blocks, with a total duration of $2 \mathrm{~h}$. For each period, NGC 1566 was observed for $28 \mathrm{~min}$, and the median system temperatures were $T_{\mathrm{sys}}=149$ and $184 \mathrm{~K}$.

The observations were centered on the nucleus, with a single-pointing field of view (FoV) equal to $18^{\prime \prime}$; the extended configuration provided in Band 7 a beam of $0.64^{\prime \prime} \times 0.43^{\prime \prime}$, with a PA of $123^{\circ}$. The galaxy was observed in dual polarization mode with $1.875 \mathrm{GHz}$ total bandwidth per baseband, and velocity resolution of $0.49 \mathrm{MHz} \sim 0.4 \mathrm{~km} \mathrm{~s}^{-1}$. The spectra were then smoothed to $11.7 \mathrm{MHz}\left(10.15 \mathrm{~km} \mathrm{~s}^{-1}\right)$ to build channel maps.
The correlator configuration was constrained by the allowed spacing of the intermediate frequencies, and therefore the lines could not be centered in their bandwidths. Although the bandwidth provided almost $1600 \mathrm{~km} \mathrm{~s}^{-1}$ each, the lines were centered at 220-250 $\mathrm{km} \mathrm{s}^{-1}$ from the edge for $\mathrm{HCN}$ and $\mathrm{CO}$, and $440 \mathrm{~km} \mathrm{~s}^{-1}$ for $\mathrm{HCO}^{+}$. Since the total line-width at zero intensity is less than $400 \mathrm{~km} \mathrm{~s}^{-1}$, this constraint has no impact on the data. We stress that we cannot have missed any outflow similar to what have been already detected in the literature (for instance that in NGC 1266, Alatalo et al. 2011): these flows are roughly symmetric in velocity, and should appear complete on the side of full velocity coverage, and also as a truncated emission on the other side of the band, which we do not see.

The total integration time provided an $\mathrm{rms}$ of $0.05 \mathrm{mJy} / \mathrm{beam}$ in the continuum, and $\sim 1.3 \mathrm{mJy} / \mathrm{beam}$ in the line cubes at $10 \mathrm{~km} \mathrm{~s}^{-1}$ resolution. Flux calibration was done on Neptune, after subtracting the line contribution from its spectrum; bandpass calibration used J1924-292, and the phase calibrations the nearby radio source J0455-462.

The maps were made with Briggs weighting and a robustness parameter of 0.5 , i.e. a trade-off between uniform and natural weighting. The data were cleaned using a mask made from the integrated $\mathrm{CO}(3-2)$ map. The continuum was subtracted from all line maps. The final cubes are $256 \times 256$ pixels of $0.11^{\prime \prime}$ per pixel in the plane of the sky, and 50 channels of $\sim 10 \mathrm{~km} \mathrm{~s}^{-1}$ width. The data were calibrated with the CASA software (v4.1; McMullin et al. 2007), and the cleaning and imaging were then finalized with the GILDAS software (Guilloteau \& Lucas 2000).

The maps and images shown in the figures of the present paper are not corrected for primary beam attenuation. However, the integrated spectra include primary beam correction, and the total masses are computed with the correction applied. Almost no $\mathrm{CO}(3-2)$ emission was detected outside the full width at halfpower (FWHP) primary beam. Due to missing short spacings, extended emission was filtered out at scales larger than $\sim 3^{\prime \prime}$ in each channel map. The elongated features detected at each velocity are, however, quite narrow spatially, thinner than $2^{\prime \prime}$, and the loss of flux may not be severe at any velocity, as can be seen in Fig. 2.

\section{Results}

Figure 2 displays 42 of the $\mathrm{CO}(3-2)$ channel maps, with a velocity range of $416 \mathrm{~km} \mathrm{~s}^{-1}$ and a velocity resolution of $10.15 \mathrm{~km} \mathrm{~s}^{-1}$. The velocity field is rather regular, showing the expected spider diagram, although slightly perturbed by spiral structure in the SE and NW directions (see also Fig. 3).

\subsection{Molecular gas distribution and morphology}

To measure fluxes we used a clipped cube where all pixel values $<2 \sigma(2.6 \mathrm{mJy} /$ beam $)$ were set to zero. The mean intensity is plotted in the lower panel of Fig. 3. Since the galaxy is more extended than the primary beam of $18^{\prime \prime}$, it is difficult to quantify the missing flux. We compare with the central spectrum obtained with a single dish in Sect. 3.4, and conclude that we might miss roughly half of the total. These single dish observations were obtained with the SEST in $\mathrm{CO}(2-1)$ with a $22^{\prime \prime}$ beam. The angular size is comparable to our FoV, but assumptions have to be made about the $\mathrm{CO}$ line ratios.

The $\mathrm{CO}$ emission is concentrated in a nuclear disk of radius $3^{\prime \prime}$ or $150 \mathrm{pc}$, where it shows a contrasted 2-arm spiral structure. The molecular gas does not seem to follow the nuclear 


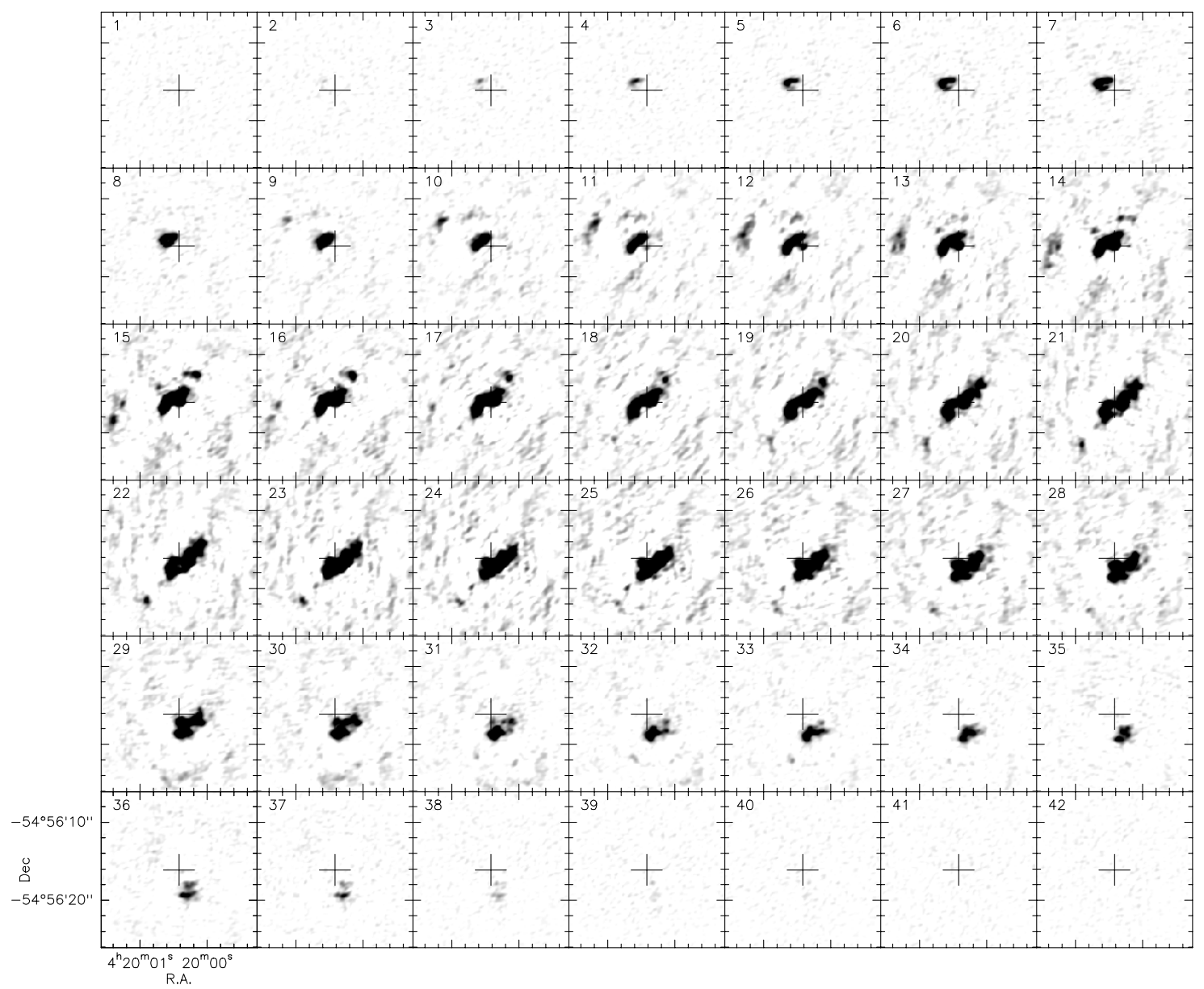

Fig. 2. Channel maps of $\mathrm{CO}(3-2)$ emission in the center of NGC 1566. Each of the 42 panels is $20^{\prime \prime}$ in size. Channels are separated by $10.15 \mathrm{~km} \mathrm{~s}^{-1}$. They are plotted from $V_{\text {hel }}=1302$ (top left) to $1718.15 \mathrm{~km} \mathrm{~s}^{-1}$ (bottom right). The synthesized beam is $0.64^{\prime \prime} \times 0.43^{\prime \prime}\left(\mathrm{PA}=123^{\circ}\right)$. The phase center of the observations (marked by a 4" cross) is given in Table 1 . The grey scale is linear, between 1 and $30 \mathrm{mJy} / \mathrm{beam}$. The central channels are saturated to better show the extent of the emission.

bar. This spiral structure at a $\sim 100 \mathrm{pc}$ scale appears independent from the large-scale grand design 2-arm structure, starting at its pseudo-nuclear ring of $\sim 35^{\prime \prime}=1.7 \mathrm{kpc}$ in radius (Elmegreen \& Elmegreen 1990). We superposed the CO map onto HST images in the $U, B, V$ and $I$ filters $(\mathrm{F} 275 \mathrm{~W}, \mathrm{~F} 450 \mathrm{~W}, \mathrm{~F} 606 \mathrm{~W}$, $\mathrm{F} 814 \mathrm{~W}$ ). All show a remarkable similarity in morphology, with the dust extinction features progressively less contrasted, as displayed in Fig. 4. The CO emission in the open spiral arms nicely corresponds to the dust lanes.

These two small spiral arms show a faint extension with a progressively smaller pitch angle, winding up onto a pseudo-ring of radius $\sim 9^{\prime \prime}=430 \mathrm{pc}$. The ring is at the edge of the FoV, and is therefore stronger in reality than it appears in our map. This ring might correspond to the inner Lindblad resonance of the nuclear bar (e.g. Buta \& Combes 1996). Such a ring has also been identified by Comeron et al. (2010) at $9.7^{\prime \prime}=465$ pc radius from the HST images, and corresponds to weak star forming regions in ionized gas (Comte \& Duquennoy 1982; Agüero et al. 2004). The main star-forming ring is, however, at the end of the bar, at about $1.5 \mathrm{kpc}$ (Elmegreen \& Elmegreen 1990).

\subsection{Continuum emission}

Continuum emission was detected at $0.87 \mathrm{~mm}$, using all four sub-bands, once the lines were subtracted. The bandwidth available then amounts to $6500 \mathrm{MHz}$, yielding an rms noise level of
$0.055 \mathrm{mJy}$. Figure 5 displays the $\mathrm{CO}(3-2)$ contours superposed on the continuum map. The $\sim 3 \mathrm{mJy}$ peak emission is detected at $50 \sigma$ significance. The emission is extended, and follows the 2-arm spiral structure already detected in the $\mathrm{CO}(3-2)$ line. The dust emission has already been detected at many wavelengths in this galaxy, with IRAS, ISO, and Spitzer. It peaks at $102 \mathrm{Jy}$ at $160 \mu \mathrm{m}$ (Dale et al. 2007), and is still $9.1 \mathrm{Jy}$ at $500 \mu \mathrm{m}$ (Wiebe et al. 2009). The emission we see at $870 \mu \mathrm{m}$ is thus expected to come essentially from dust. From the radio emission at $\mathrm{cm}$ wavelengths, the slope of the synchrotron emission can be derived to be -0.8 . Extrapolating from the measured point at $5 \mathrm{GHz}$, the total synchrotron emission is expected to be $3 \mathrm{mJy}$. On the high frequency side, the extrapolation of a grey-body, with a dust opacity $\propto v^{2}$, gives a flux at 870 microns of $1 \mathrm{Jy}$. The dust emission is then dominating the flux at 870 microns.

The total continuum emission integrated over our FoV amounts to $12.5 \mathrm{mJy}$, which is consistent with the extrapolation of the far-infrared SED from previous measurements, if account is taken for the missing flux and the continuum extent: the total flux for the whole galaxy should be on the order of $1 \mathrm{Jy}$. Most of the emission must come from outside the ALMA FoV, and it is likely that the interferometer misses a significant part of the emission, more so than for the line where the velocity splitting reduces the extent of the emission, in each channel.

The central peak in the continuum emission, coinciding with the peak in the $\mathrm{CO}(3-2)$ emission at the center of the nuclear 


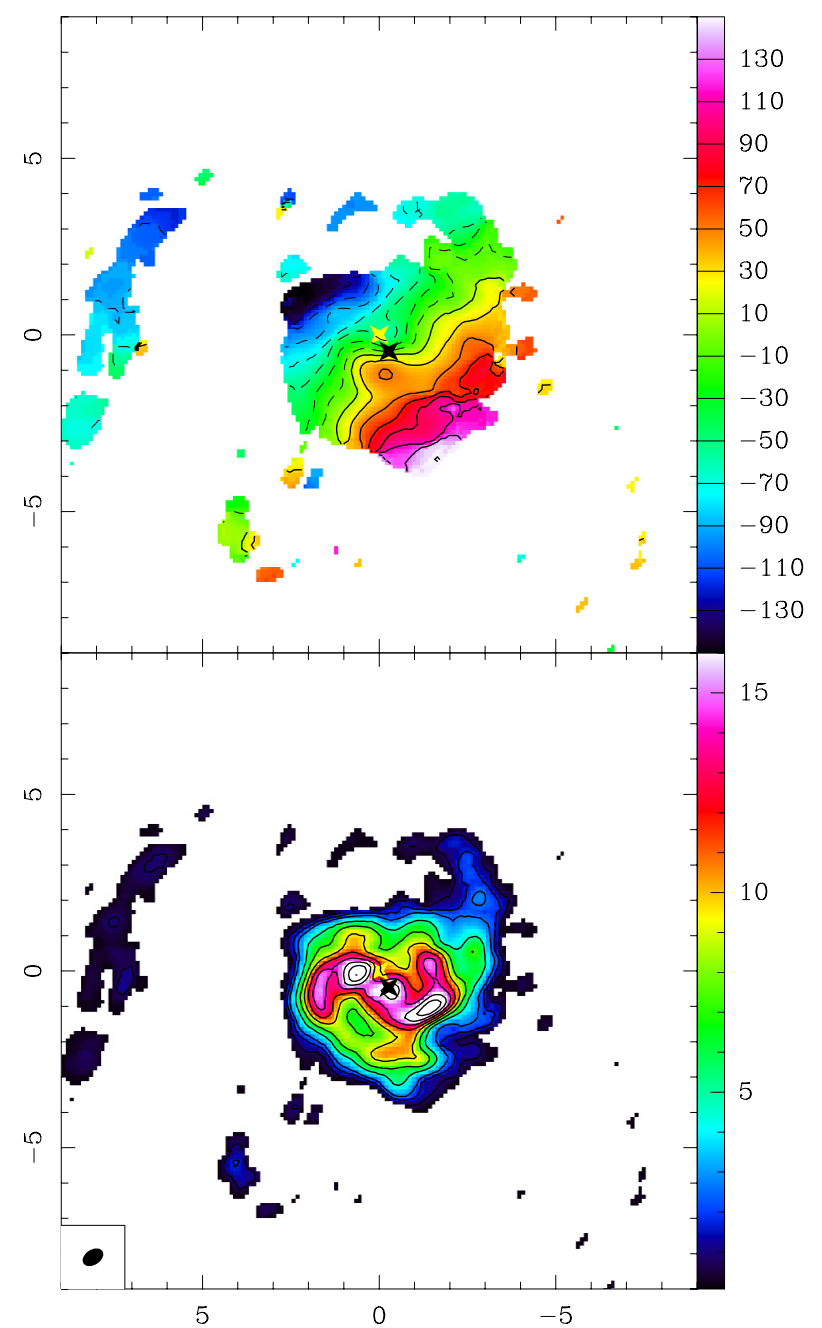

Fig. 3. Velocity field (top) and integrated intensity (bottom) of the $\mathrm{CO}(3-2)$ emission in the center of NGC 1566. Coordinates are in arcsec relative to the phase center of Table 1 . The latter is indicated as a yellow cross, and the new adopted center as a black cross (see Sect. 3.2). The color palettes are in $\mathrm{km} \mathrm{s}^{-1}$ relative to $V_{\mathrm{hel}}=1504 \mathrm{~km} \mathrm{~s}^{-1}$ (top) and in $\mathrm{Jy} /$ beam $\times \mathrm{MHz}\left(\right.$ or $0.87 \mathrm{Jy} /$ beam $\times \mathrm{km} \mathrm{s}^{-1}$ ) at the bottom. The beam size of $0.64^{\prime \prime} \times 0.43^{\prime \prime}$ is indicated at the bottom left.

spiral arm, is adopted as the new center of the galaxy (cf. Table 1). The distance between the two centers is $\sim 0.5^{\prime \prime}=24 \mathrm{pc}$, and might result from the uncertainty of the optical position because of dust patches at the center.

\subsection{CO kinematics}

The top panel of Fig. 3 displays the velocity field of the molecular gas. The velocity field is well described by rotation, with the major axis at a position angle of $44^{\circ}$ and an inclination of $35^{\circ}$ as determined by optical studies at larger scales. The $\mathrm{CO}$ velocities reveal a peak in the rotation curve at about $2^{\prime \prime}$, then a slight decrease to reach a plateau, similar to what is found in optical studies. Although the $\mathrm{CO}$ rotation curve shown in Fig. 9 seems to be poorly constrained by the scattered $\mathrm{CO}$ data points, it was more precisely derived by minimizing the residuals in the 2D map (see below).

The $\mathrm{H} \alpha$ rotation curve was determined by Agüero et al. (2004) from 6 slits of width 2 '. 8 that have insufficient spatial resolution to resolve the $2^{\prime \prime}$ peak. In addition, there is a deficiency

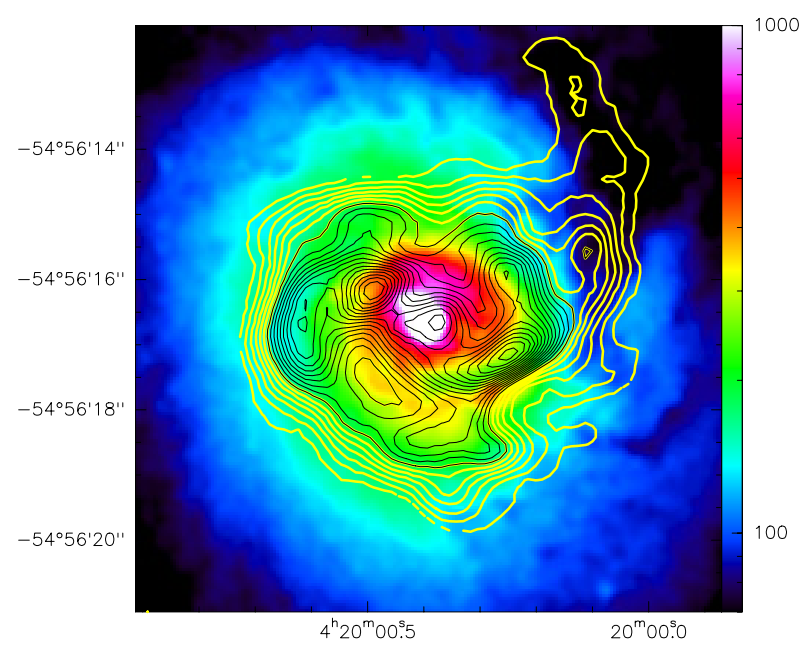

Fig. 4. Overlay of $\mathrm{CO}(3-2)$ contours on the (F606W) HST image. The color scale is linear, in arbitrary units. Notice the perfect coincidence between the emergent spiral arm in $\mathrm{CO}(3-2)$ and the dust extinction in the NW. CO contours are linear from 0.04 to 17.44 in steps of $0.87 \mathrm{Jy} /$ beam $\mathrm{km} \mathrm{s}^{-1}$. The first contours of the $\mathrm{CO}(3-2)$ emission are drawn in yellow to be visible against the dark dust lane.

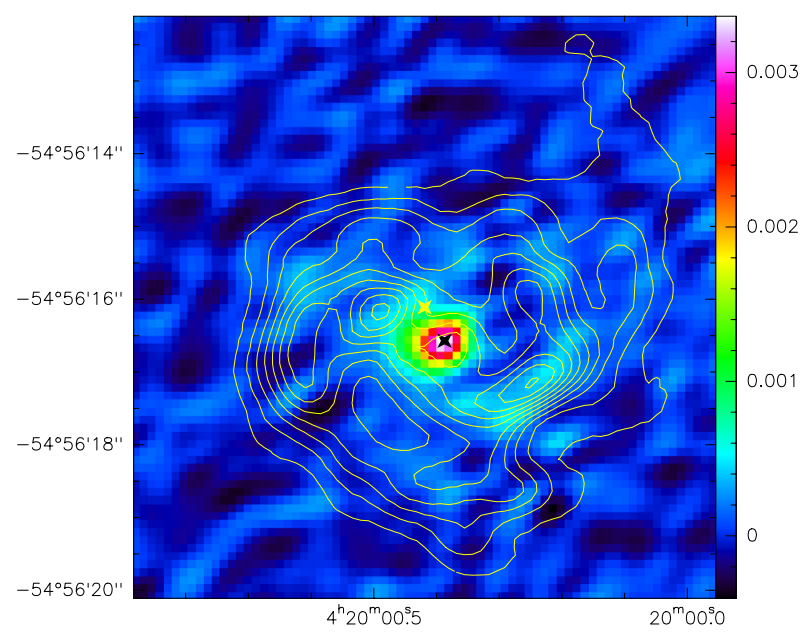

Fig. 5. Overlay of $\mathrm{CO}(3-2)$ contours (as in Fig. 4) on the $0.87 \mathrm{~mm}$ continuum image. The field of view is $8^{\prime \prime}$ in diameter. The yellow cross shows the phase center, while the black cross defines the new center adopted in Table 1 . The color scale is in Jy/beam.

of HII regions inside a radius of $22^{\prime \prime}=1 \mathrm{kpc}$, corresponding to the pseudo ring where the grand design spiral arms begin. The Fabry-Perot $\mathrm{H} \alpha$ rotation curves from Comte \& Duquennoy (1982) and Pence et al. (1990) also suffer from this HII region deficiency, and are consistent with our curve only at larger radii. The model $\mathrm{H} \alpha$ rotation curve from Agüero et al. (2004) shown in Fig. 9 follows a linear curve inside the ring, as expected for any tracer distribution with a hole at the center. The main star forming ring appears at the end of the bar, and might correspond to the corotation of the bar, coinciding with the inner Lindblad resonance of the spiral (Elmegreen \& Elmegreen 1990). This would explain the deficiency of ionized gas inside $1 \mathrm{kpc}$ radius. Therefore, only the CO gas can probe the disk inside the stellar bar. The deficiency of star formation in these regions emitting molecular lines might be due to the dynamical state of the gas, which disfavors star formation, like in NGC 1530 for example (Reynaud \& Downes 1998). 


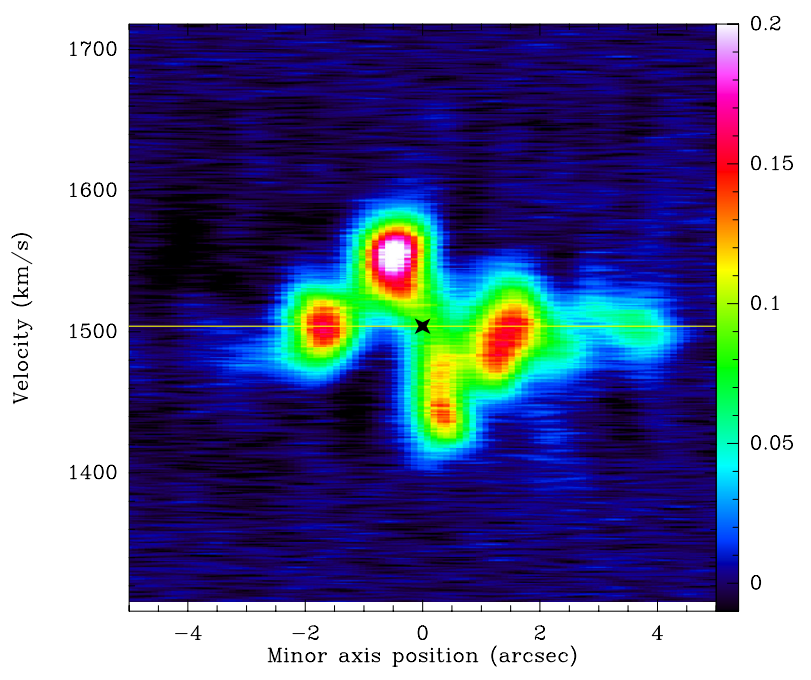

Fig. 6. $\mathrm{CO}(3-2)$ position-velocity diagram along the minor axis at $\mathrm{PA}=$ $134^{\circ}$ (SE is at left, and is the far side). Note the small deviations from a constant radial velocity, which come from spiral streaming motions. The black cross indicates the adopted center.

The stellar bar is mainly visible in red and near-infrared images, inside the star forming ring. The molecular gas does not follow the bar, but instead develops a nuclear spiral structure, which reflects some kinematical perturbations. These are most visible in the position-velocity diagram along the minor axis (Fig. 6), while the rotation is more regular along the major axis (Fig. 7). These kinematic disturbances arise more from gaseous spiral and turbulent perturbations than from coherent streaming motions due to a bar. This situation is similar to that in other galaxies in the NUGA sample, which show similar wiggles on the minor axis PV diagram but remain regular on the major axis: NGC 4826 (García-Burillo et al. 2003), NGC 3147 (Casasola et al. 2008) or NGC 1961 (Combes et al. 2009). In NGC 1566, the bar has a position angle of nearly $0^{\circ}$, significantly different from the major axis position angle of $44^{\circ}$. This is a favorable situation for measuring streaming motions due to the bar. However, the gas follows its own spiral structure that is more aligned with the major axis, explaining why little perturbation shows up along this symmetry axis.

Another way to reveal peculiar velocities in $2 \mathrm{D}$ over the nuclear region is to subtract the expected regular velocity field derived from the $\mathrm{CO}$ data from the velocity field. Figure 8 displays the residuals obtained relative to the adopted rotation curve model of Fig. 9. They are very low all over the map, and peak at $\sim 30 \mathrm{~km} \mathrm{~s}^{-1} \mathrm{~S}$-SE of the nucleus, corresponding to the edge of an arm. Since at large-scale trailing spiral structures are always dominant, the observed velocity field indicates that the SE region is the far side, and the NW the near side. Streaming motions are stronger on the far side, but are present on both sides, while following the spiral arms. This pattern is not characteristic of a symmetric outflow of gas. It has the right sign for an outflow, since it is redshifted on the far side of the minor axis and blueshifted on the near side, but it has much too small an amplitude. Note also that at larger scales, Agüero et al. (2004) find the opposite, i.e. an inflow of gas along the minor axis (see their Fig. 3), with a comparable amplitude. This supports our interpretation in terms of streaming motions. The stronger "outflow" feature coincides in size and position with the one-sided NLR ionized cone in the [OIII] high resolution image of Schmitt \& Kinney (1996), but the coincidence is most likely spurious,

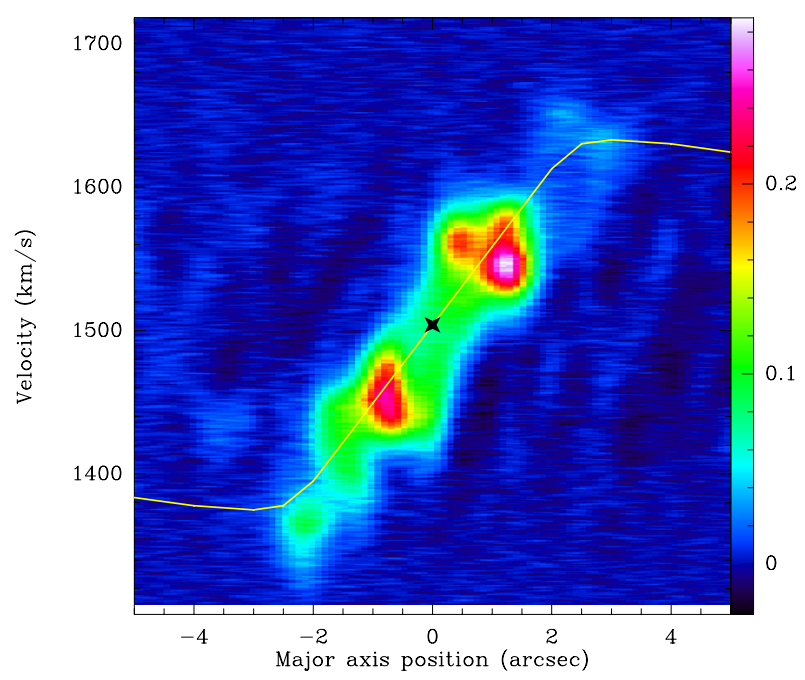

Fig. 7. $\mathrm{CO}(3-2)$ position-velocity diagram along the major axis at $\mathrm{PA}=$ $44^{\circ}$ (East is at left). The velocity profile is quite regular, and matches with the expected rotational velocity gradient (yellow line), as modeled in Fig. 9. The black cross indicates the adopted center.

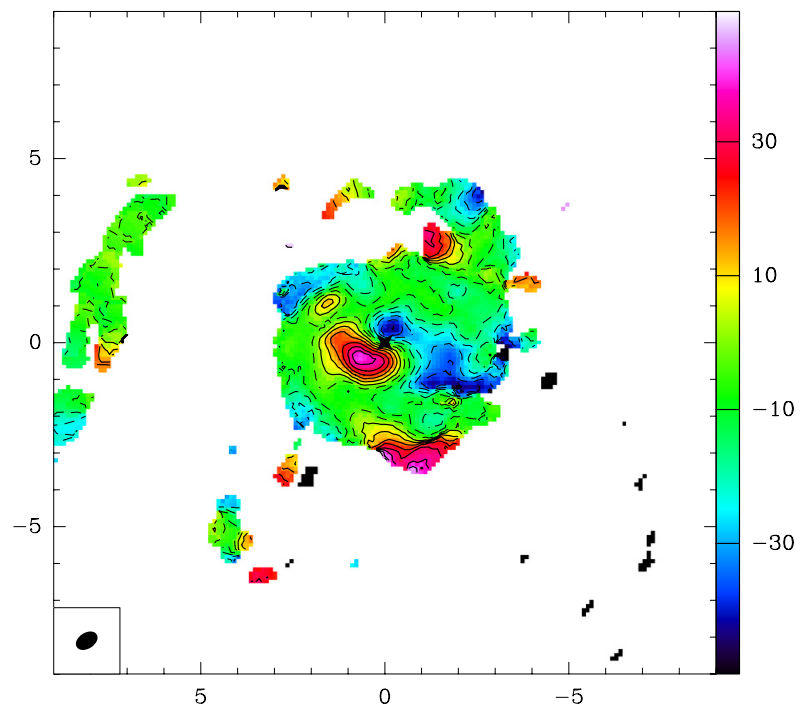

Fig. 8. Velocity residuals after subtraction of a regular rotation model, based on the CO data, as displayed in Fig. 9. The beam size of $0.64^{\prime \prime} \times$ $0.43^{\prime \prime}(30 \times 20 \mathrm{pc})$ is indicated at lower left. The black cross indicates the adopted center.

since there is no reason for the cone to be related to the spiral arm streaming motion.

\section{4. $\mathrm{H}_{2}$ mass}

Figure 10 displays the total $\mathrm{CO}(3-2)$ spectrum integrated over the observed map. When integrated over the spectral range $\left(F W H M=155 \mathrm{~km} \mathrm{~s}^{-1}\right)$, the integrated emission is $596 \pm$ $2 \mathrm{Jy} \mathrm{km} \mathrm{s}^{-1}$. Towards the central position, Bajaja et al. (1995) found a $\mathrm{CO}(2-1)$ spectrum peaking at $T_{A}^{*}=120 \mathrm{mK}$ with $F W H M=200 \mathrm{~km} \mathrm{~s}^{-1}$, yielding a total integrated flux of $660 \mathrm{Jy} \mathrm{km} \mathrm{s}^{-1}$, in a beam of $22^{\prime \prime}$. Since their beam is very similar to our FoV, the comparison is meaningful, telling us that we are certainly missing some flux, by a factor up to $\sim 2$. Indeed, the $\mathrm{CO}$ excitation appears high at the center of NGC 1566, since the $\mathrm{CO}(2-1) / \mathrm{CO}(1-0)$ intensity ratio is about 1 in temperature units, 


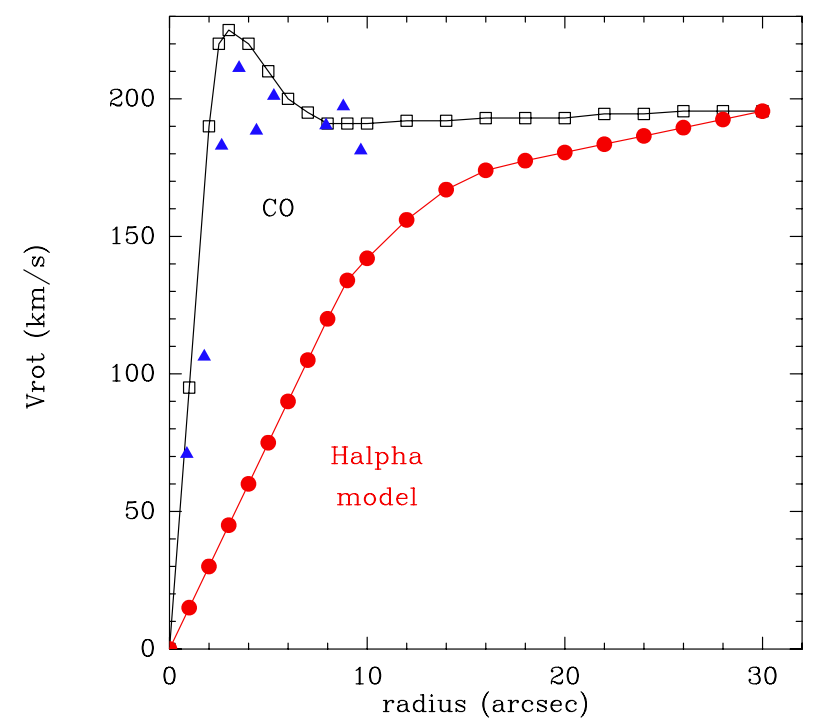

Fig. 9. Rotational velocity model adopted for NGC 1566 (black open rectangles), based on the $\mathrm{CO}$ data points (blue triangles), and minimizing the residuals of Fig. 8. The $\mathrm{H} \alpha$ kinematic model from Agüero et al. (2004) is also shown in red circles. Those data were obtained with 2 ". 8 wide slits, with insufficient spatial resolution to resolve the central peak. The $\mathrm{H} \alpha$ velocity model rises linearly because of the deficiency of HII regions inside a radius of 22" (see text).

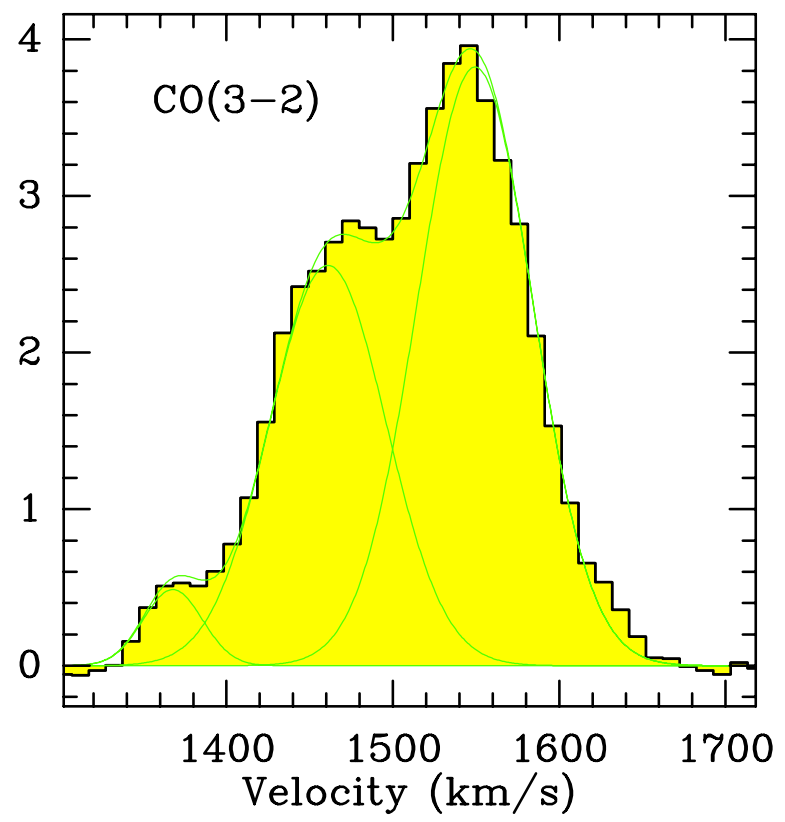

Fig. 10. Total $\mathrm{CO}(3-2)$ spectrum, integrated over the observed map, with a FoV of $18^{\prime \prime}$. The vertical scale is in Jy. The green line is the result of the Gaussian fit with 3 velocity components (see Table 2). The spectrum is primary beam corrected.

averaged over the $43^{\prime \prime}$ beam (Bajaja et al. 1995). This is expected for thermalized excitation and a dense molecular medium. In that case the $\mathrm{CO}(3-2)$ flux should be higher than the $\mathrm{CO}(2-1)$ by up to a factor of 2.2 (flux varying as $\sim v^{2}$ for gas at temperature larger than $25 \mathrm{~K}$ and density larger than $10^{4} \mathrm{~cm}^{-3}$ ). Already the $\mathrm{CO}(2-1)$ spectrum of Bajaja et al. (1995) appears broader in velocity, which indicates some missing flux in our $\mathrm{CO}(3-2)$ map. The SEST observations point to a total molecular mass of $1.3 \times 10^{9} M_{\odot}$, and in the central $43^{\prime \prime}$ beam of $3.5 \times 10^{8} M_{\odot}$. In

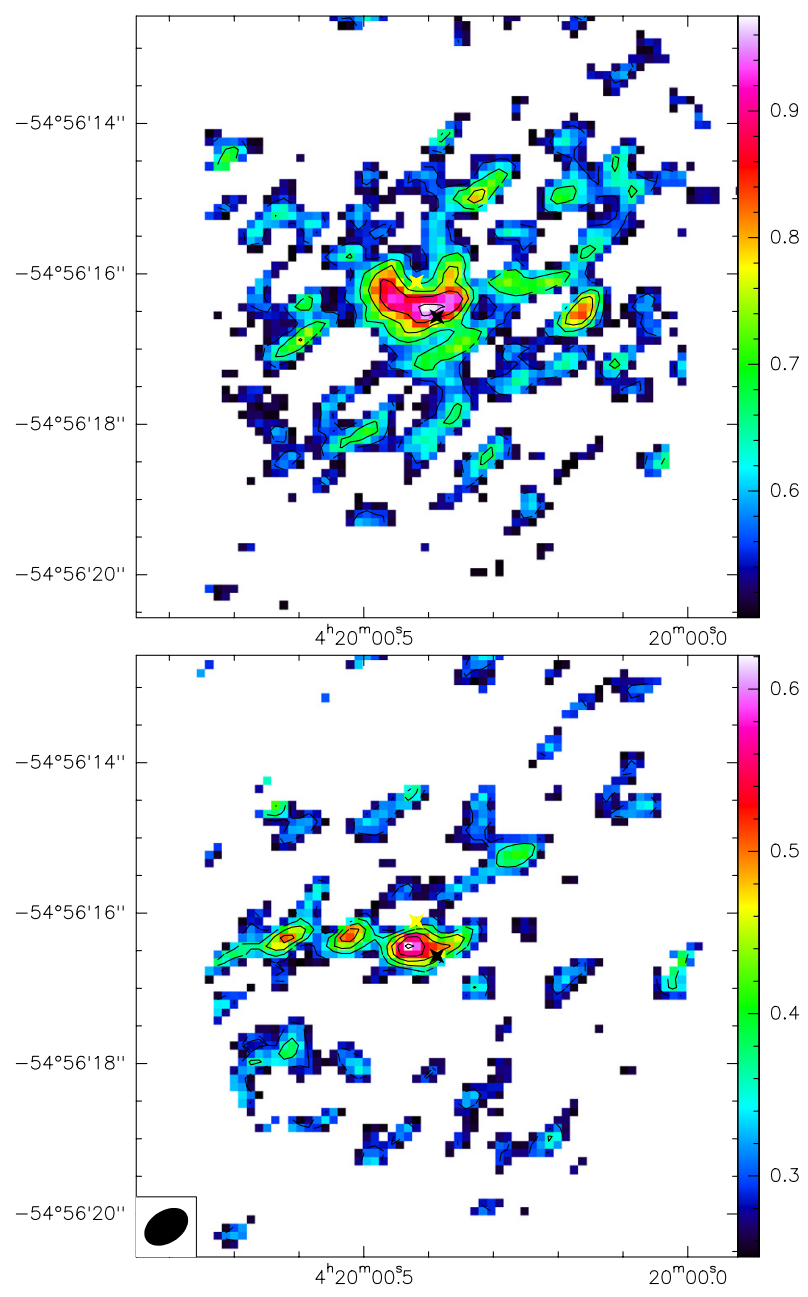

Fig. 11. Top: map of the $\mathrm{HCO}^{+}(4-3)$ line in NGC 1566. The adopted center is drawn with a black cross, and the phase center by a yellow cross. The color scale is in Jy/beam $\times \mathrm{MHz}\left(\right.$ or $\left.0.87 \mathrm{Jy} / \mathrm{beam} \times \mathrm{km} \mathrm{s}^{-1}\right)$ ) Bottom: same for $\mathrm{HCN}(4-3)$. The beam size of $30 \times 20 \mathrm{pc}$ is indicated at the lower left.

the $22^{\prime \prime}$ beam, the SEST $\mathrm{CO}(2-1)$ spectrum, together with the $\mathrm{CO}(2-1) / \mathrm{CO}(1-0)$ ratio of 1 , yields a mass $1.7 \times 10^{8} M_{\odot}$, while we find $0.7 \times 10^{8} M_{\odot}$ in our FoV of $18^{\prime \prime}$ assuming thermally excited gas, and a Milky-Way like CO-to- $\mathrm{H}_{2}$ conversion factor, of $2.3 \times 10^{20} \mathrm{~cm}^{-2} /\left(\mathrm{K} \mathrm{km} \mathrm{s}^{-1}\right)($ e.g. Solomon \& Vanden Bout 2005).

\section{5. $\mathrm{HCO}^{+}$and $\mathrm{HCN}$}

Along with $\mathrm{CO}(3-2)$, we detect the $\mathrm{HCO}^{+}(4-3)$ and $\mathrm{HCN}(4-3)$ emission lines, mainly in the center of the galaxy. The corresponding maps are displayed in Fig. 11, and the integrated spectra in Fig. 12. The lines are mainly detected in the nuclear spiral structure, and are too faint to see the rest of the nuclear disk. The $\mathrm{HCO}^{+}$line is 3 times stronger than the $\mathrm{HCN}$ line, which is expected for starburst galaxies such as M82 (e.g. Seaquist \& Frayer 2000). The detection of these lines reveals a high proportion of dense gas, since the critical densities to excite the $J=4-3$ transition of $\mathrm{HCO}^{+}$and $\mathrm{HCN}$ are $6.5 \times 10^{6}$ and $1.6 \times 10^{8} \mathrm{~cm}^{-3}$, respectively.

The integrated intensities and profile characteristics displayed in Table 2 reveal that the $\mathrm{HCO}^{+}$line width is comparable to the $\mathrm{CO}(3-2)$ width, while the $\mathrm{HCN}$ spectrum appears 

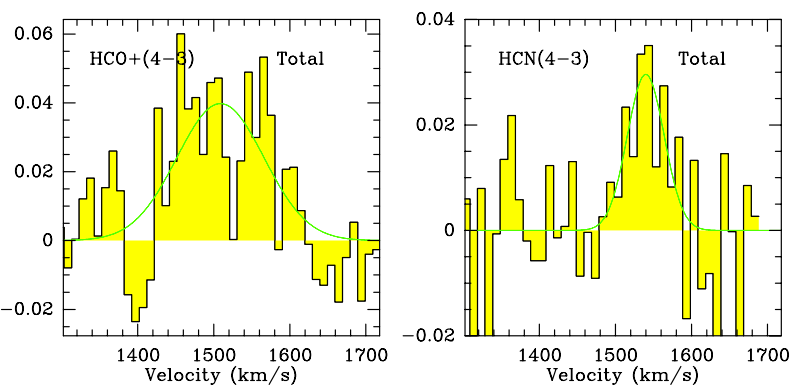

Fig. 12. Total $\mathrm{HCO}^{+}(4-3)$ (left) and $\mathrm{HCN}(4-3)$ (right) spectra, integrated over the field of view of $18^{\prime \prime}$, after primary beam correction. The vertical scale is in Jy. The green lines are the Gaussian fits, whose parameters are displayed in Table 2.

Table 2. Line fluxes, after primary beam correction.

\begin{tabular}{lcccc}
\hline \hline Line & $\begin{array}{c}\text { Area } \\
\mathrm{Jy} \mathrm{km} \mathrm{s}^{-1}\end{array}$ & $\begin{array}{c}V \\
\mathrm{~km} \mathrm{~s}^{-1}\end{array}$ & $\begin{array}{c}\Delta V^{a} \\
\mathrm{~km} \mathrm{~s}^{-1}\end{array}$ & $\begin{array}{c}\text { Flux }^{b} \\
\mathrm{Jy}\end{array}$ \\
\hline $\mathrm{CO}(3-2)$ & $596 \pm 2$ & $1516 \pm 0.3$ & $155 \pm 1$ & 3.6 \\
$\mathrm{C} 1$ & $21 \pm 1$ & $1368 \pm 1$ & $42 \pm 1$ & 0.5 \\
$\mathrm{C} 2$ & $197 \pm 5$ & $1455 \pm 1$ & $77 \pm 1$ & 2.4 \\
$\mathrm{C} 3$ & $368 \pm 5$ & $1545 \pm 1$ & $91 \pm 1$ & 3.8 \\
$\mathrm{HCO}^{+}(4-3)$ & $5.7 \pm 0.6$ & $1509 \pm 7$ & $135 \pm 14$ & 0.04 \\
$\mathrm{HCN}^{(4-3)}$ & $1.8 \pm 0.4$ & $1540 \pm 7$ & $57 \pm 14$ & 0.03 \\
\hline
\end{tabular}

Notes. Results of the Gaussian fits: first line for $\mathrm{CO}(3-2)$ assumes only one component; following lines assume 3 velocity components $(\mathrm{C} 1, \mathrm{C} 2$ and C3), shown in Fig. 10. ${ }^{(a)}$ Full width at half maximum (FWHM). (b) Peak flux.

truncated, as if only one side of the galaxy, the redshifted SW part, is strong enough to be detected. The total intensities of both lines are, however, weak: $\mathrm{HCO}^{+}(4-3)$ is 100 times weaker than $\mathrm{CO}(3-2)$, and $\mathrm{HCN}(4-3)$ is 300 times weaker than $\mathrm{CO}(3-2)$. These ratios are about 3 times less than at the center of M 82, and suggest a lower fraction of dense gas (e.g. Naylor et al. 2010). This is expected since NGC 1566 is not a starburst galaxy (e.g., Gao \& Solomon 2004).

The fact that the $\mathrm{HCO}^{+}$emission is 3 times stronger than the $\mathrm{HCN}$ emission indicates that the excitation of the molecules at the center of NGC 1566 is dominated by star formation (PDR or photodissociation region), and not the AGN (XDR or $\mathrm{X}$-ray dominated region). Indeed, in AGN-dominated molecular disks, the $\mathrm{HCN} / \mathrm{HCO}^{+}$ratio can reach values much larger than 1 (Kohno et al. 2003; Krips et al. 2008; García-Burillo et al. 2010; Costagliola et al. 2011; Imanishi \& Nakanishi 2013).

\section{Torques and AGN fueling}

A small bar is detected in red and near-infrared images of NGC 1566 (see for example Fig. 1 from Agüero et al. 2004, from 2MASS). Its position angle is $\mathrm{PA}=0^{\circ}$, and its length is $35^{\prime \prime}=1.7 \mathrm{kpc}$, as determined by Hackwell \& Schweizer (1983), or Comeron et al. (2010). According to Elmegreen \& Elmegreen (1990), this radius coincides with kinks in the spiral arms, and the amplitude of the conspicuous 2-arm grand design spiral begins to increase. We have used a red image (F814W) from HST to derive the stellar potential in the center, since 2MASS images have insufficient angular resolution. We have not separated the bulge from the disk contribution since NGC 1566 is a late type (Sbc) galaxy. This means that the bulge was effectively

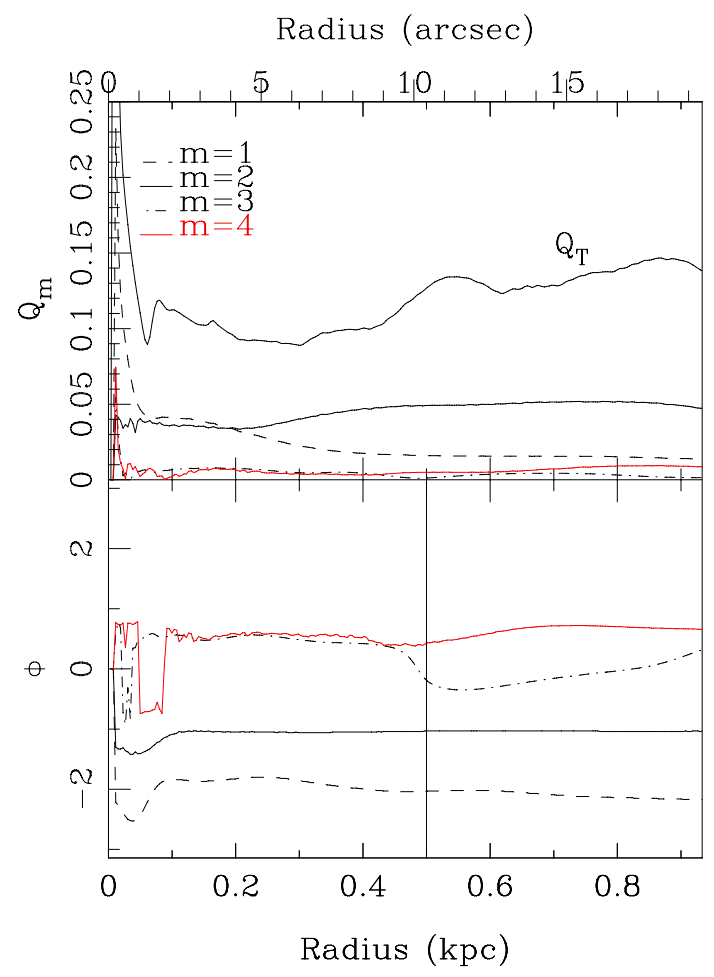

Fig. 13. Top: strengths $\left(\mathrm{Q}_{m}\right.$ and total $\left.\mathrm{Q}_{T}\right)$ of the $m=1$ to $m=4$ Fourier components of the stellar potential within the central kpc. The $m=2$ term is dominant, and has a constant phase, corresponding to the bar. Bottom: corresponding phases in radians of the Fourier components, taken from the major axis, in the deprojected image. The vertical line indicates the proposed ILR position for the nuclear bar.

assumed to be flattened. Dark matter can be safely neglected inside the central kpc. The image has been rotated and deprojected according to $\mathrm{PA}=44^{\circ}$ and $i=35^{\circ}$, and then Fourier transformed to compute the gravitational potential and forces. A stellar exponential disk thickness of $\sim 1 / 12$ th of the radial scalelength of the galaxy $\left(h_{\mathrm{r}}=3.8 \mathrm{kpc}\right)$ has been assumed, giving $h_{\mathrm{z}}=317 \mathrm{pc}$. This is the average scale ratio for galaxies of this type (e.g., Barteldrees \& Dettmar 1994; Bizyaev \& Mitronova $2002,2009)$. The potential has been obtained assuming a constant mass-to-light ratio of $M / L=0.5 M_{\odot} / L_{\odot}$ in the $I$-band over the considered portion of the image of $2 \mathrm{kpc}$ in size. This value is realistic in view of what is found statistically for spiral galaxies (Bell \& de Jong 2001). The pixel size of the map is $0.079^{\prime \prime}=3.8 \mathrm{pc}$. The stellar $M / L$ value was fit to reproduce the observed CO rotation curve. We have checked that the results are not significantly changed when the geometrical parameters ( $i$ and PA) are varied by $\pm 5^{\circ}$, as found in the literature. The $M / L$ varies as $1 / \sin ^{2} i$ accordingly.

The potential $\Phi(R, \theta)$ can be decomposed into its different Fourier components:

$\Phi(R, \theta)=\Phi_{0}(R)+\sum_{m} \Phi_{m}(R) \cos \left(m \theta-\phi_{m}(R)\right)$.

The strength of the $m$-Fourier component, $Q_{m}(R)$ is defined as $Q_{m}(R)=m \Phi_{m} / R\left|F_{0}(R)\right|$, i.e. by the ratio between tangential and radial forces (e.g. Combes \& Sanders 1981). The strength of the total non-axisymmetric perturbation $Q_{T}(R)$ is defined similarly with the maximum amplitude of the tangential force $F_{T}^{\max }(R)$. Their radial distributions and the radial phase variations are displayed in Fig. 13. 


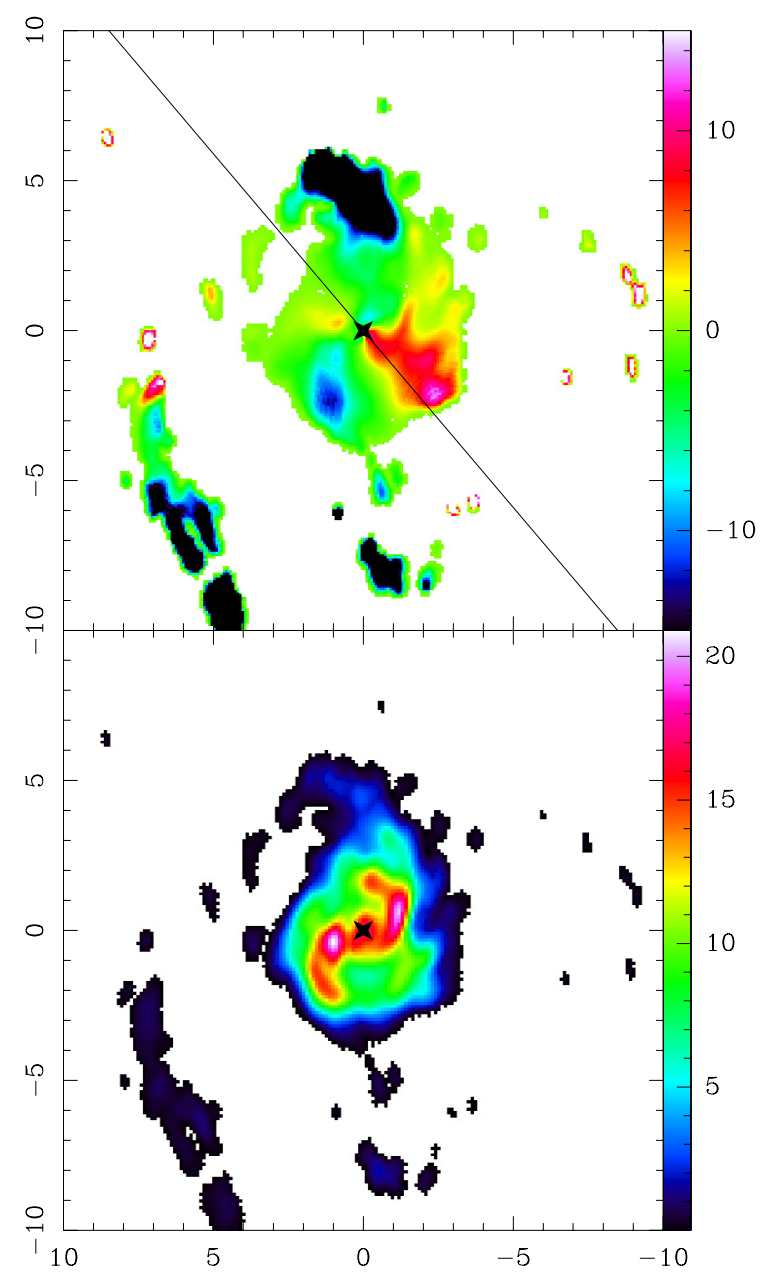

Fig. 14. Top: map of the gravitational torque, $(t(x, y) \times \Sigma(x, y)$, as defined in text) in the center of NGC 1566. The torques change sign as expected in a four-quadrant pattern (or butterfly diagram). The orientation of the quadrants follows the nuclear bar's orientation. In this deprojected picture, the major axis of the galaxy is oriented parallel to the horizontal axis. The inclined line reproduces the mean orientation of the bar ( $\mathrm{PA}=40^{\circ}$ on the deprojected image). Bottom: deprojected image of the $\mathrm{CO}(3-2)$ emission, at the same scale, and with the same orientation, for comparison. The axes are labeled in arcsecond relative to the center. The color scales are linear, in arbitrary units.

The derivatives of the potential yield the forces per unit mass $\left(F_{x}\right.$ and $\left.F_{y}\right)$ at each pixel, and the torques per unit mass $t(x, y)$ are then computed by:

$t(x, y)=x F_{y}-y F_{x}$.

The sign of the torque is determined relative to the sense of rotation in the plane of the galaxy. The product of the torque and the gas density $\Sigma$ at each pixel allows one then to derive the net effect on the gas, at each radius. This quantity $t(x, y) \times \Sigma(x, y)$, is shown in Fig. 14.

The torque weighted by the gas density $\Sigma(x, y)$ is then averaged over azimuth, i.e.

$t(R)=\frac{\int_{\theta} \Sigma(x, y) \times\left(x F_{y}-y F_{x}\right)}{\int_{\theta} \Sigma(x, y)}$.

The quantity $t(R)$ represents the time derivative of the specific angular momentum $L$ of the gas averaged azimuthally (e.g.

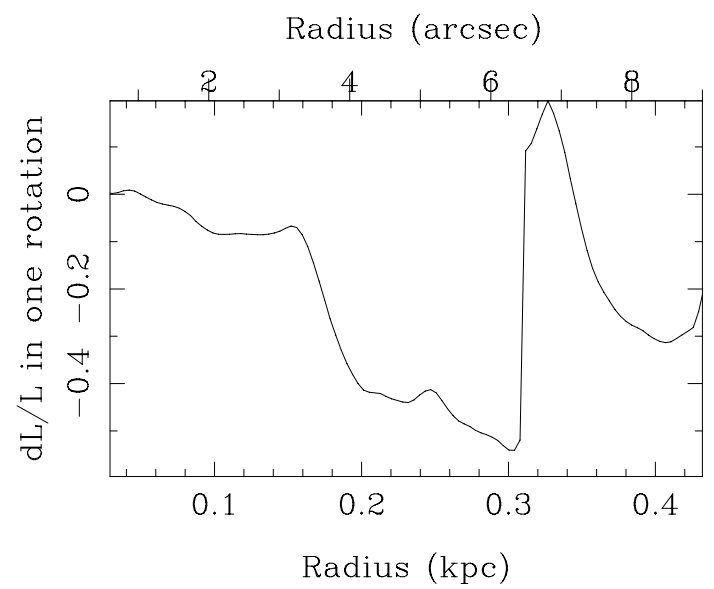

Fig. 15. Radial distribution of the torque, quantified by the fraction of the angular momentum transferred from the gas in one rotation- $\mathrm{d} L / L$, estimated from the $\mathrm{CO}(3-2)$ deprojected map. The forces at each point of the map were computed from the $I$-band HST image (see text). The torque is negative inside a $0.3 \mathrm{kpc}$ radius and down to the spatial resolution of the observations. Between 6 and $9^{\prime \prime}$ from the center, there is not much $\mathrm{CO}$ emission and the torque estimation is noisy.

García-Burillo et al. 2005). Normalizing at each radius by the angular momentum and rotation period allows us to estimate the efficiency of the gas flow, as shown in Fig. 15.

Although the nuclear bar strength is moderate, Fig. 15 shows that its fueling efficiency is high. Between 200 and 300 pc, the gas loses half of its angular momentum in one rotation, which is $\sim 7 \mathrm{Myr}$. Inside $100 \mathrm{pc}$, the relative loss rate is lower, but other dynamical phenomena can then contribute, such as dynamical friction of molecular clouds against the distribution of stars. The rotation period becomes smaller than $3 \mathrm{Myr}$, and the dynamical friction time-scale is decreasing rapidly, especially for massive giant molecular clouds. Since dense clouds exist at the center of NGC 1566, as supported by the $\mathrm{HCN}$ and $\mathrm{HCO}^{+}$detections, this might be the mechanism for driving the gas down to $10 \mathrm{pc}$ scales, in times comparable to a few rotation periods. The dynamical friction time-scale $t_{\mathrm{df}}$ at a radius $R$ can be estimated in a galaxy like the Milky Way as $t_{\mathrm{df}}(\mathrm{Myr})=(\mathrm{R} / 10 \mathrm{pc})^{2}\left(10^{6} M_{\odot} / M_{\text {cloud }}\right)$ for a molecular cloud of mass $M_{\text {cloud }}$ (e.g., Combes 2002).

As shown in Fig. 14, the nuclear spiral structure inside the nuclear ILR ring of the bar is of a trailing nature and is located inside the negative torque quadrants. This might appear suprising, since in many cases the spiral structure is predicted to be leading in this region, and the torque positive, maintaining the gas in the ILR ring (e.g., Buta \& Combes 1996). However, in the presence of a sufficiently massive black hole, this behavior can be reversed: the spiral becomes trailing, and the torque negative. Indeed, the indicator of the precession rate of elliptical orbits in the frame of the epicyclic approximation, $\Omega-\kappa / 2$, is significantly modified by a central massive body. Instead of decreasing regularly towards zero at the center, the $\Omega-\kappa / 2$ curve increases steeply as $r^{-3 / 2}$. The gas undergoes collisions, loses energy and spirals progressively towards the center. When the precession rate decreases, the series of elliptical orbits precess more and more slowly, and then lag at smaller radii, forming a leading structure. When the precession rate increases, they form a trailing structure.

Another question is whether the orbits change orientation at the ILR, as expected if the $x_{2}$ orbits develop between two inner resonances. This would destroy the nuclear bar and be observable on the infrared image. The lower panel of Fig. 13 shows the 


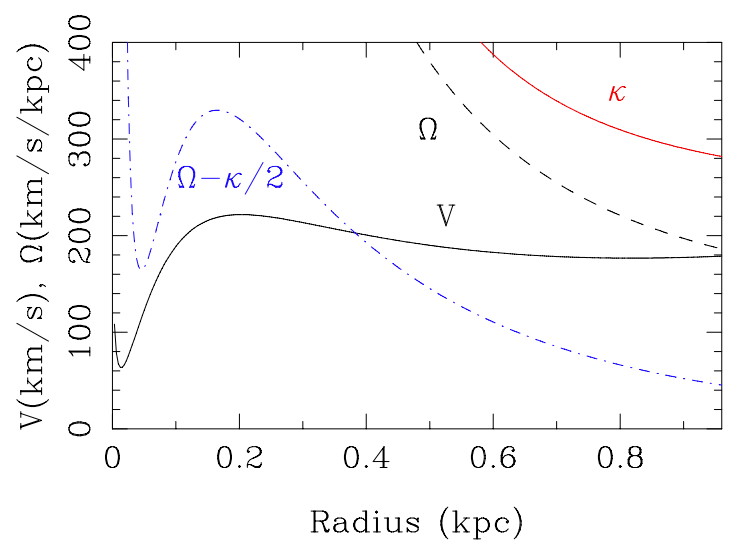

Fig. 16. Schematic representation of the rotation curve (black) epicyclic frequency $\kappa$ (red), and corresponding $\Omega-\kappa / 2$ curve (blue) within the central kpc of NGC 1566. The contribution of a super-massive black hole in the nucleus with $M_{\mathrm{BH}}=8.3 \times 10^{6} M_{\odot}$ has been included.

phases of the various Fourier components. The bar $(m=2)$ has a constant phase over its length, and their orientation change does not appear to be significant.

NGC 1566 is therefore an example of a trailing spiral inside the nuclear ring of a bar i.e., the case described in Fig. 79b of Buta \& Combes (1996). This means that the mass of the black hole should be sufficiently high to have an influence on the gas dynamics on a $100 \mathrm{pc}$ scale. Woo \& Urry (2002) have estimated a BH mass of $M_{\mathrm{BH}}=8.3 \times 10^{6} M_{\odot}$ from the $\mathrm{M}-\sigma$ relation using a measured central velocity dispersion (Table 1 ). With this plausible black hole mass, we have estimated the $\mathrm{BH}$ influence. Although the modification of the rotation curve is very small, the influence on the precession rate $\Omega-\kappa / 2$ is more significant. Figure 16 illustrates more clearly how this is possible.

In this figure, we selected an analytical form for the rotation curve that schematically reproduces the observed one. It is not possible to make a better fit, given that only the $\mathrm{CO}$ emission is able to trace the kinematics in this region. If we locate corotation at the end of the bar, i.e. at a radius of $1.7 \mathrm{kpc}$, then the pattern speed of the bar is $\sim 120 \mathrm{~km} \mathrm{~s}^{-1} / \mathrm{kpc}$. The ILR is then approximately at a radius of $500 \mathrm{pc}$, near the location where we found the resonant ring (see Sect. 3.1 and also Comeron et al. 2010). This is a good fit, given that the computation of resonances via the $\Omega-\kappa / 2$ curve and the epicyclic approximation is not accurate. The precession rate of the infalling orbits is then going to increase towards the center, accounting for the trailing spiral structure observed.

Figure 16 shows that the influence of the black hole on the rotation curve is almost negligible; it is significant only inside $13 \mathrm{pc}$. However, it is more important in influencing the precession rate, which includes the second derivative of $\Omega$. The influence of the $\mathrm{BH}$ is then felt up to $50 \mathrm{pc}$. Since the $100 \mathrm{pc}$-scale arms are defined by elongated orbits extending below $50 \mathrm{pc}$, it is possible that the black hole indeed has an influence on the 2-arm spiral structure inside the ILR ring.

\subsection{Comparison with other nuclear patterns}

The observation of a trailing 2-arm spiral structure inside the ILR of a nuclear bar is a rare configuration. In molecular gas morphologies, this case had not previously been encountered. Although previous NUGA studies have not had the advantage of as small a physical resolution as the current ALMA data, it is useful to make some statistical considerations. Looking at previous NUGA galaxies where detailed torques have been measured, it is possible to distinguish at least three generic configurations (see also García-Burillo \& Combes 2012):

1. There is no ILR, and the trailing structure goes right to the nucleus from corotation (CR). A trailing spiral structure is then expected throughout, with negative torques (as in NGC 3147, 3627). In some cases, like NGC 6574, there is an ILR, but it cannot be resolved with the CO beam; negative torques are measured from CR to ILR. So this case is also similar to the no-ILR case.

2. There is an ILR, and a ring or pseudo-ring at the ILR, which is resolved by the molecular observations. The molecular gas reveals a hole inside the ring, or is highly deficient inside that radius. The torque is positive inside the ILR (negative outside), so the gas is piling up in the ring. When there is some $\mathrm{CO}$ emission inside the ring, its distribution is in general patchy, with no clear arms; it could be a mixture of leading and trailing features, but there must be some leading pattern to account for positive torques (this is the case for NGC 4321, 4569, 5248, 5953, 6951 and 7217). NGC 1433 is also in this configuration, although the nuclear spiral structure is rather flocculent (Combes et al. 2013). The spiral is trailing between CR and ILR of the nuclear bar, and within the ILR at $200 \mathrm{pc}$ in radius, the spiral structure is unclear, but the torque is positive.

3. There is an independent embedded structure, a nuclear bar with a different pattern speed from that of the primary bar, and a negative torque can be measured inside the CR of the nuclear bar, but without a coherent orientation (case of NGC 2782).

There are also other special cases, e.g. involving development of a dominant $m=1$ pattern in the center, like NGC 1961, 4826, or 5850 .

Another way to detect spiral structure in the nuclear gas is to amplify the dust extinction features in the HST images through unsharp masking. Martini et al. (2003a,b) have studied the presence of circumnuclear dust in 123 galaxies, of which 14 have nuclear rings. When circumnuclear dust features are present, they have classified them as grand design 2-arm structures (GD), multi-arm or floculent structures (tightly or loosely wound) and chaotic structures (spiral or not). Only 19 galaxies have GD nuclear morphologies, and among the nuclear ring galaxies, only 3 are classified as GD (NGC 1300, 3081 and 6890). Only galaxies with large scale bars exhibit GD nuclear spiral structure. Most of these spiral structures continue the dust lanes along the bar, without any ILR. In their sample of 75 nearby galaxies, Peeples \& Martini (2006) find 7 objects with GD nuclear spirals. Only two have both a large-scale grand design and a GD, NGC 3081 and 5643. In these galaxies, however, the sense of winding of the dust spirals in the very center is unclear, and there could be a mixture of leading and trailing features. A clear case of a trailing spiral inside a nuclear ring is still missing. We have also examined the compilation of nuclear rings of Comeron et al. (2010). Most rings are either devoid of structure, or display confused or chaotic dust structures. Although the nuclear ring is clearly seen in the NGC 1566 image, the interior structure is difficult to distinguish in dust maps, making the molecular gas emission a better tracer.

\section{Summary}

We have presented ALMA cycle 0 results for a Seyfert 1 galaxy from our extended NUGA sample, NGC 1566. The observations 
in $\mathrm{CO}(3-2)$ allow us to reach a superb spatial resolution of $25 \mathrm{pc}$, even in this early cycle.

The center of NGC 1566 has a dense nuclear disk of molecular gas, that is deficient in both atomic gas and HII regions. The central disk is typically $\sim 3.5^{\prime \prime}=170 \mathrm{pc}$ in radius, and reveals an open 2-arm nuclear spiral structure, which tends to wind up into a faint ring at $430 \mathrm{pc}$ radius. The spiral structure coincides very well with obscured dust lanes in HST images, and also with dust continuum emission at $870 \mu \mathrm{m}$. The nuclear spiral arms are dense enough to be detected in the $\mathrm{HCO}^{+}(4-3)$ and $\mathrm{HCN}(4-3)$ lines.

The kinematics of the $\mathrm{CO}$ emission show a rather regular rotational velocity field, with only slight perturbations from the 2-arm nuclear spiral. There are in particular redshifted streaming motions on the SE part of the minor axis, and blueshifted ones on the NW side, along the border of the spiral arms. However, the amplitude of these perturbations is small, and it is remarkable that no symmetric molecular outflow, indicating a possible AGN feedback, is observed.

The faint $\mathrm{CO}$ ring at $r=430 \mathrm{pc}$, which is more strongly noticeable in the $V-I$ color HST image (Comeron et al. 2010), is plausibly stronger in reality given the reduced sensitivity at the edge of our FoV. This ring corresponds to the ILR of the bar, if corotation is assumed to coincide with the bar end. The trailing spiral observed in $\mathrm{CO}$ emission is then well inside the ILR ring of the bar. We have computed the gravitational potential from the stars within the central kpc, from the I-band HST image. Weighting the torques on each pixel by the gas surface density observed in the $\mathrm{CO}(3-2)$ line has allowed us to estimate the sense of the angular momentum exchange and its efficiency. The gravity torques are negative from 50 to $300 \mathrm{pc}$. Between $200 \mathrm{pc}$ and $300 \mathrm{pc}$, the gas loses half of its angular momentum in a rotation period. Gravity torques are then very efficient in fueling the AGN, and bring molecular clouds so close to the center that dynamical friction could drive them to the nucleus in a few Myr.

This work provides the first example of a clear trailing spiral observed in molecular gas inside the ILR ring of a bar, indicating that the super-massive black hole is influencing the gas dynamics significantly enough to reverse gravity torques. Instead of maintaining the ILR ring density, the torques are then driving the gas towards the nucleus, a first step towards possibly fueling the AGN.

Acknowledgements. The ALMA staff in Chile and ARC staff at IRAM are gratefully acknowledged for their help in the data reduction. We want to thank in particular Gaelle Dumas and Philippe Salomé for their useful advice. This paper makes use of the following ALMA data: ADS/JAO.ALMA\#2011.0.00208.S. ALMA is a partnership of ESO (representing its member states), NSF (USA) and NINS (Japan), together with NRC (Canada) and NSC and ASIAA (Taiwan), in cooperation with the Republic of Chile. The Joint ALMA Observatory is operated by ESO, AUI/NRAO and NAOJ. The National Radio Astronomy Observatory is a facility of the National Science Foundation operated under cooperative agreement by Associated Universities, Inc. We used observations made with the NASA/ESA Hubble Space Telescope, and obtained from the Hubble Legacy Archive, which is a collaboration between the Space Telescope Science Institute (STScI/NASA), the Space Telescope European Coordinating Facility (ST-ECF/ESA), and the Canadian Astronomy Data Centre (CADC/NRC/CSA). F.C. acknowledges the European Research Council for the Advanced Grant Program Num 267399-Momentum. I.M. acknowledges financial support from the Spanish grant AYA2010-15169 and from the Junta de Andalucia through TIC-114 and the Excellence Project P08-TIC-03531. We made use of the NASA/IPAC Extragalactic Database (NED), and of the HyperLeda database.

\section{References}

Abramowicz, M. A., Xu, C., \& Lasota, J. P. 1986, Proc. IAU Symp., 119, 371 Agüero E. L., Diaz R. J., \& Bajaja, E. 2004, A\&A 414, 453

Alatalo, K., Blitz, L., Young, L. M., et al. 2011, ApJ, 735, 88
Alloin, D., Pelat, D., Phillips, M. M., \& Whittle M., 1985, ApJ, 288, 205 Alloin, D., Pelat, D., Phillips, M. M., Fosbury, R. A. E., \& Freeman, K. 1986 ApJ, 308, 23

Bajaja, E., Wielebinsli, R., Reuter, H. P., et al. 1995, A\&AS, 114, 147

Baribaud, T., Alloin, D., Glass, I., \& Pelat, D. 1992, A\&A, 256, 375

Barteldrees, A., \& Dettmar, R.-J. 1994, A\&AS, 103, 475

Bell, E. F., \& de Jong, R. S. 2001, ApJ, 550, 212

Buta, R., \& Combes, F. 1996, Fund. Cosm. Phys., 17, 95

Bizyaev, D., \& Mitronova, S. 2002, A\&A, 389, 795

Bizyaev, D., \& Mitronova, S. 2009, ApJ, 702, 1567

Casasola, V., Combes, F., Garcna-Burillo, S., et al. 2008, A\&A, 490, 61

Comeron, S., Knapen, J. H., Beckman, J. E., et al. 2010, MNRAS, 402, 2462

Combes, F., \& Sanders, R. H. 1981, A\&A, 96, 164

Combes, F. 2002, in 7th cosmology colloquium, High Energy Astrophysics, for and from Space [arXiv: astro-ph/0208113]

Combes, F., Baker, A. J., Schinnerer, E., et al. 2009, A\&A 503, 73

Combes, F., García-Burillo, S., Casasola, V., et al. 2013, A\&A 558, A124

Comte, G., \& Duquennoy, A. 1982, A\&A, 114, 7

Costagliola, F., Aalto, S., Rodriguez, M. I., et al. 2011, A\&A, 528, A30

Croton, D. J., Springel, V., White, S. D. M., et al. 2006 MNRAS, 365, 11

Dale, D. A., Gil de Paz, A., Gordon, K. D., et al. 2007, ApJ, 655, 863

Di Matteo, T., Colberg, J., Springel, V., Hernquist, L., \& Sijacki, D. 2008, ApJ, 676,33

Elmegreen, B. G., \& Elmegreen, D. M., 1990, ApJ, 355, 52

Feruglio, C., Maiolino, R., Piconcelli, E., et al. 2010, A\&A, 518, L155

Gao, Y., \& Solomon, P. M. 2004, ApJS, 152, 63

Garcìa-Burillo, S., Combes, F., Hunt, L. K., et al. 2003, A\&A, 407, 485

Garcìa-Burillo, S., Combes, F., Schinnerer, E., Boone, F., \& Hunt, L.K. 2005, A\&A, 441, 1011

Garcìa-Burillo, S., Usero, A., Fuente, A., et al. 2010, A\&A, 519, A2

Garcìa-Burillo, S., \& Combes, F. 2012, J. Phys. Conf. Ser., 372, 012050

Glass, I. S. 2004, MNRAS, 350, 1049

Guilloteau, S., \& Lucas, R. 2000, in Imaging at Radio through Submillimeter Wavelengths, ASP Conf. Proc., 217, 299

Gültekin, K., Richstone, D. O., Gebhardt, K., et al. 2009, ApJ, 698, 198

Hackwell, J. A., \& Schweizer, F. 1983 ApJ, 265, 643

Harnett, J. I. 1984, MNRAS, 210, 13

Harnett, J. I. 1987, MNRAS, 227, 887

Ho, L. C., Li, Z-Y, Barth, A. J., et al. 2011, ApJS, 197, 21

Hopkins, P., Hernquist, L., Cox, T. J., et al. 2006, ApJS, 163, 1

Hopkins, P. F., \& Quataert, E. 2010, MNRAS, 407, 1529

Imanishi, M., \& Nakanishi, K. 2013, AJ, 146, 47, 91

Jogee, S. 2006 Lect. Notes Phys., 693, 143

Korchagin, V., Kikuchi, N., Miyama, S. M., et al. 2000, ApJ, 541, 565

Kilborn, V. A., Koribalski, B. S., Forbes, D., et al. 2005, MNRAS, 356, 77

Kohno, K, Ishizuki, S., Matsushita, S., et al. 2003, PASJ, 55, L1

Krips, M., Neri, R., García-Burillo, S., et al. 2008, ApJ, 677, 262

Krips, M., Martyn, S., Eckart, A., et al. 2011, ApJ, 736, 37

Levenson, N. A., Radomski, J. T., Packham, C., et al. 2009, ApJ, 703, 390

Malkan, M., Gorjian, V., \& Tam, R. 1998, ApJS, 117, 25

Martini, P., Regan, M. W., Mulchaey, J. S., \& Pogge, R. W. 2003a, ApJS, 146, 353

Martini, P., Regan, M. W., Mulchaey, J. S., \& Pogge, R. W. 2003b, ApJ, 589, 774

McMullin, J. P., Waters, B., Schiebel, D., Young, W., \& Golap, K. 2007, in Astronomical Data Analysis Software and Systems XVI, ASP Conf. Ser., 376,127

Naylor, B. J., Bradford, C. M., Aguirre, J. E., et al. 2010, ApJ, 722, 668

Pence, W. D., Taylor, K., \& Atherton, P. 1990, ApJ, 357, 415

Peeples, M. S., \& Martini, P. 2006, ApJ, 652, 1097

Reif, K., Mebold, U., Goss, W. M., et al. 1982, A\&AS, 50, 451

Renaud, F., Bournaud, F., Emsellem, E., et al. 2013, MNRAS, 436, 1836

Reunanen, J., Kotilainen, J. K., \& Prieto, M. A. 2002, MNRAS, 331, 154

Reunanen, J., Prieto, M. A., \& Siebenmorgen, R. 2010, MNRAS, 402, 879

Reynaud, D., \& Downes, D. 1998, A\&A, 337, 671

Riffel, R. A., \& Storchi-Bergmann, T. 2011, MNRAS, 411, 469

Rupke, D. S., Veilleux, S., \& Sanders, D. B. 2005, ApJ, 632, 751

Schmitt, H., \& Kinney, A. 1996, ApJ, 463, 498

Seaquist, E. R., \& Frayer, D. T. 2000, ApJ, 540, 765

Shlosman, I., Frank, J., \& Begelman, M. C. 1989, Nature, 338, 45

Sijacki, D., Springel, V., Di Matteo, T., \& Hernquist, L. 2007, MNRAS, 380, 877

Solomon, P. M., \& Vanden Bout, P. A. 2005, ARA\&A, 43, 677

Sturm, E., Gonzalez-Alfonso, E., Veilleux, S., et al. 2011, ApJ, 733, L16

Wiebe, D. V., Ade, P. A. R., Bock, J. J., et al. 2009, ApJ, 707, 1809

Woo, J.-H., Urry, C. M. 2002, ApJ, 579, 530 